H. Urakawa

Nagoya Math. J.

Vol. 78 (1980), 137-152

\title{
ON THE LEAST POSITIVE EIGENVALUE OF THE LAPLACIAN FOR THE COMPACT QUOTIENT OF A CERTAIN RIEMANNIAN SYMMETRIC SPACE
}

\author{
HAJIME URAKAWA
}

\section{§1. Introduction and statement of results}

Let $(\tilde{M}, g)$ be the standard Euclidean space or a Riemannian symmetric space of non-compact type of rank one. Let $G$ be the identity component of the Lie group of all isometries of $(\tilde{M}, g)$. Let $\Gamma$ be a discrete subgroup of $G$ acting fixed point freely on $\tilde{M}$ whose quotient manifold $M_{\Gamma}$ is compact. Let $-\Delta_{\Gamma}$ be the Laplace-Beltrami operator (cf. [4]) acting on smooth functions on $M_{\Gamma}$ for the Riemannian metric $g_{\Gamma}$ on $M_{\Gamma}$ induced by $g$. The compactness of $M_{\Gamma}$ implies that the spectrum of $\Delta_{\Gamma}$ forms a discrete subset of the set of non-negative real numbers. Let $\lambda_{1}(T)$ be the least positive eigenvalue of $\Delta_{\Gamma}$. Let $\operatorname{vol}\left(M_{\Gamma}\right)$ be the volume of $\left(M_{\Gamma}, g_{\Gamma}\right)$. Then we have

Theorem A. Let $(\tilde{M}, g)$ be the $n$-dimensional standard Euclidean space, so that $\left(M_{\Gamma}, g_{r}\right)$ is a compact flat manifold. Then we have

$$
\lambda_{1}(\Gamma) \operatorname{vol}\left(M_{\Gamma}\right)^{2 / n} \leqq n^{-1}(2+n)^{1+2 / n}\left[\frac{2 \pi^{n / 2}}{\Gamma(n / 2)}\right]^{2 / n}\left[j_{n / 2-1}\right]^{2-4 / n},
$$

where the number $j_{n / 2-1}$ is the least positive zero point of the $(n / 2-1)$-th Bessel function $J_{n / 2-1}$.

Remark. Since $j_{n / 2-1} \sim n / 2$ as $n \rightarrow \infty$ (cf. [7] p. 153), the right hand side of $(1)$ is $(\pi \mathrm{e} / 2) n=(4.2699 \cdots) n$ asymptotically as $n \rightarrow \infty$.

Let $\mu_{n}=\max _{\Gamma} \lambda_{1}(\Gamma) \operatorname{vol}\left(M_{\Gamma}\right)^{2 / n}$ where the maximum is taken over all lattices $\Gamma$ of $\boldsymbol{R}^{n}$. For a lattice $\Gamma$ of $\boldsymbol{R}^{n}$, the spectrum of the corresponding flat torus $\left(M_{\Gamma}, g_{\Gamma}\right)$ is given by $\left\{4 \pi^{2}|x|^{2} ; x \in \Gamma^{*}\right\}$, where $\Gamma^{*}$ is a dual lattice of $\Gamma,|x|^{2}=(x, x), x \in \boldsymbol{R}^{n}$ and ( , ) is the inner product of $\boldsymbol{R}^{n}$ which gives the standard Riemannian metric on $\boldsymbol{R}^{n}$ (cf. [1]). So we have $\lambda_{1}(\Gamma)$

Received September 18, 1978.

Revised September 10, 1979. 
$=4 \pi^{2} \min _{x \in \Gamma^{*-(0)}}|x|^{2}$. On the other hand, vol $\left(M_{\Gamma}\right)=\operatorname{det}\left(\Gamma^{*}\right)^{-1 / 2}$ (cf. [6]). Here $\operatorname{det}\left(\Gamma^{*}\right)$ is the determinant of the matrix $\left(\left(b_{i}, b_{j}\right)\right)_{1 \leqq i, j \leqq n}$, where $\left\{b_{i}\right\}_{i=1}^{n}$ is a basis of $\boldsymbol{R}^{n}$ generating the lattice $\Gamma^{*}$. Then the above $\mu_{n}$ coincides $4 \pi^{2}$ times the largest possible value for the ratio

$$
\mu\left(\Gamma^{*}\right)=\left(\min _{x \in \Gamma^{*}-(0)}|x|^{2}\right)\left(\operatorname{det}\left(\Gamma^{*}\right)\right)^{-n}
$$

where $\Gamma^{*}$ varies over all lattices in $\boldsymbol{R}^{n}$. A problem to compute the value $\mu_{n}$ for every $n$ is related to the following classical problem (cf. [6] p. 34): What is the maximum possible density for a union of non-overlapping balls of fixed radius in $\boldsymbol{R}^{n}$ ? But until now the value $\mu_{n}$ is unknown for $n \geqq 9$. In 1905, H. Minkowski has given (cf. [6]) a lower estimate for $\mu_{n}$ by

$$
\mu_{n}>4 \pi^{2} \omega_{n}^{-2 / n}
$$

where $\omega_{n}$ is the volume of the unit disk in $R^{n}$ and $4 \pi^{2} \omega_{n}^{-2 / n} \sim(2 \pi / e) n=$ $(2.3115 \cdots) n$ as $n \rightarrow \infty$. On the other hand, in 1958, C. A. Rogers has given (cf. [6]) an upper estimate for $\mu_{n}$ by

$$
\mu_{n} \leqq Q_{n}
$$

where the constant $Q_{n}$ is $(4 \pi / \mathrm{e}) n=(4.6229 \ldots) n$ asymptotically as $n \rightarrow \infty$. The above remark implies that Theorem A improves the result of Rogers in the asymptotic sense.

Theorem B. Let $(\tilde{M}, g)$ be a Riemannian symmetric space of noncompact type of rank one. Let $G$ be the connected component of the Lie group of all isometries of $(\tilde{M}, g)$. We normalize $g$ in such a way that it is induced by the Killing form of the Lie algebra of $G$. Consider all discrete subgroups $\Gamma$ of $G$ acting fixed point freely on $\tilde{M}$ whose quotient manifold $M_{\Gamma}$ is compact. Then we have

$$
\lim _{\operatorname{vol}\left(M_{\left.\Gamma^{\prime}\right) \rightarrow \infty}\right.} \sup _{1}(\Gamma) \leqq|\delta|^{2},
$$

for a positive constant $|\delta|^{2}$ depending only on $(\tilde{M}, \mathrm{~g})$ (cf. $\left.\S 2\right)$.

Notice that every real valued zonal spherical function $\varphi_{2}$ on $\tilde{M}$ corresponding to the principal series of $G$ (cf. [10]) satisfies (cf. [3])

$$
\Delta \varphi_{\lambda}=\left(|\lambda|^{2}+|\delta|^{2}\right) \varphi_{\lambda}, \quad|\lambda|^{2} \geqq 0 .
$$

Here $-\Delta$ is the Laplace-Beltrami operator of $(\tilde{M}, g)$ and it satisfies (cf. § 4 ) $\Delta(f \circ \pi)=\left(\Delta_{\Gamma} f\right) \circ \pi$ for every smooth function $f$ on $M_{\Gamma}$, where $\pi$ is the natural 
projection of $\tilde{M}$ onto $M_{\Gamma}$. If $(\tilde{M}, g)$ is the unit disc with the Poincare metric, then Theorem B has been obtained by H. Huber [5].

I would like to express my thanks to Professor T. Sunada for his advices during the preparation of this paper.

\section{§2. Preliminaries}

In this section, following [2] and [3], we prepare some properties of the zonal spherical functions on the Euclidean space or a Riemannian symmetric space of non-compact type of rank one.

2.1. Let $(\tilde{M}, g)$ be the standard Euclidean space $\left(\boldsymbol{R}^{n}, g\right)$. Let $\left(x_{1}, \cdots\right.$, $\boldsymbol{x}_{n}$ ) be the orthonormal coordinate of $\boldsymbol{R}^{n}$. Let $-\Delta=\sum_{i=1}^{n} \partial^{2} / \partial x_{i}^{2}$ be the Laplace-Beltrami operator on $\boldsymbol{R}^{n}$. The zonal spherical functions on $\boldsymbol{R}^{n}$ (cf. [9]) are eigen-functions of $\Delta$ depending only on $r=|x|, x \in \boldsymbol{R}^{n}$, whose values at 0 are 1 . For example (cf. [7]), for $p \in \boldsymbol{R}(p>0)$, consider the functions

$$
\Phi_{p}(x)= \begin{cases}\Gamma\left(\frac{n}{2}\right)\left(\frac{p r}{2}\right)^{1-n / 2} J_{n / 2-1}(p r) & (x \neq 0) \\ 1 & (x=0)\end{cases}
$$

Then $\Phi_{p}$ is real analytic on $\boldsymbol{R}^{n}$ and written as $\Phi_{p}(x)=\Psi_{p}(r)$ where $\Psi_{p}(s)$ $=\psi(p s)$ and $\psi$ is an even function on $\boldsymbol{R}$ defined by

$$
\psi(s)=\Gamma\left(\frac{n}{2}\right) \sum_{m=0}^{\infty}(-1)^{m}(m !)^{-1} \Gamma\left(\frac{n}{2}+m\right)^{-1}\left(\frac{s}{2}\right)^{2 m} .
$$

Then $\Psi_{p}$ satisfies the equation

$$
-\frac{d^{2}}{d r^{2}} \Psi_{p}-\frac{n-1}{r} \frac{d}{d r} \Psi_{p}=p^{2} \Phi_{p} .
$$

Recalling the general equality:

$$
\Delta F=-\frac{\partial^{2}}{\partial r^{2}} F-\frac{n-1}{r} \frac{\partial F}{\partial r}
$$

for a rotationary invariant function $F \in C^{2}\left(R^{n}-(0)\right)$, we get $\Delta \Phi_{p}=p^{2} \Phi_{p}$ (cf. [9]). Let $j_{n / 2-1}$ be the least positive zero point of $J_{n / 2-1}$. Let $f$ be the even function on $R$ by 


$$
f(s)= \begin{cases}\Psi_{p}(s)^{1+\varepsilon}, & |s| \leqq \frac{j_{n / 2-1}}{p}, \\ 0, & |s| \geqq \frac{j_{n / 2-1}}{p},\end{cases}
$$

where $0<\varepsilon<1$. Then $f$ satisfies

LEMMA 2.1. (1) $f$ belongs to $C^{1}(R)$ and the support of $f$ is contained in the set $\left\{|s| \leqq j_{n / 2-1} \mid p\right\}$, (2) $f^{\prime \prime}$ is continuous except the point $|s|=j_{n / 2-1} / p$, (3) $f^{\prime \prime}(s)=O\left(|| s\left|-j_{n / 2-1} / p\right|^{\varepsilon-1}\right)$, so $f^{\prime \prime} \in L^{1}(R)$, and (4) $L_{1}(f)+(1+\varepsilon) p^{2} f \geqq 0$ $\left(|s| \neq 0, j_{n / 2-1} / p\right)$, for the differential operator $L_{1}$ on $R-(0)$ defined by

$$
L_{1}=\frac{d^{2}}{d s^{2}}+\frac{n-1}{s} \frac{d}{d s} .
$$

Proof. (1) and (2) are clear. (3) is due to the fact that the number $j_{n / 2-1}$ is the zero point of $J_{n / 2-1}$ of first order (cf. [7] p. 151). By (2.1), we have

$$
L_{1}(f)+(1+\varepsilon) p^{2} f=(1+\varepsilon) \varepsilon\left(\frac{d \Psi_{p}}{d s}\right)^{2} \Psi_{p}^{\varepsilon-1} \geqq 0
$$

for $0<|s|<j_{n / 2-1} / p$, so (4) holds.

Q.E.D.

Let $F$ be the function on $\boldsymbol{R}^{n}$ defined by $F(x)=f(|x|), x \in \boldsymbol{R}^{n}$. Then we have

Lemma 2.2. $F$ belongs to $C^{1}\left(\boldsymbol{R}^{n}\right)$ and $C^{2}\left(\boldsymbol{R}^{n}-\gamma_{1}\right)$ where $\gamma_{1}=\left\{x \in \boldsymbol{R}^{n}\right.$; $|x|=0$ or $\left.j_{n / 2-1} \mid p\right\}$, and the support of $F$ is contained in the set $\left\{x \in \boldsymbol{R}^{n}\right.$; $\left.|x| \leqq j_{n / 2-1} / p\right\}$. Moreover

$$
\begin{gathered}
(\Delta F)(x)=-\left(L_{1} f\right)(|x|) \quad(x \neq 0) \text { and } \Delta F \in L^{1}\left(\boldsymbol{R}^{n}\right), \\
\Delta F \leqq(1+\varepsilon) p^{2} F \quad \text { on } \boldsymbol{R}^{n}-\gamma_{1} .
\end{gathered}
$$

Proofs are immediate from Lemma 2.1.

Due to Lemma 2.1, there exists a sequence $\left\{f_{m}\right\}_{m=1}^{\infty}$ of smooth even functions on $R$ such that (5) $f_{m}(s)=f(s) \quad\left(|s| \leqq j_{n / 2-1} / 2 p\right)$ and $f_{m}(s)=0$ $\left(|s| \leqq 2 j_{n / 2-1} / p\right)$, (6) $f_{m}$ (resp. $f_{m}^{\prime}$ ) converges to $f$ (resp. $f^{\prime}$ ) uniformly on $R$ as $m \rightarrow \infty$ and $(7) \lim _{m \rightarrow \infty} \int_{-\infty}^{\infty}\left|f_{m}^{\prime \prime}(s)-f^{\prime \prime}(s)\right| d s=0$.

Define $F_{m} \in{\stackrel{m \rightarrow \infty}{C}\left(\boldsymbol{R}^{n}\right)}^{\infty}$ by $F_{m}(x)=f_{m}(|x|), x \in \boldsymbol{R}^{n}$. Then by (5), (6) and (7), the support of $F_{m}$ is included in the set $\left\{x \in R^{n} ;|x| \leqq 2 j_{n / 2-1} / p\right\}, F_{m}$ converges to $F$ uniformly on $\boldsymbol{R}^{n}$ and 


$$
\lim _{m \rightarrow \infty} \int_{R^{n}}\left|\Delta F_{m}-\Delta F\right| d x=0
$$

where $d x$ is the Lebesgue measure on $\boldsymbol{R}^{n}$.

2.2. Let $(\tilde{M}, g)$ be a Riemannian symmetric space of non-compact type of rank one. Let $G$ be the identity component of the Lie group of all isometries of $(\tilde{M}, g)$. Let $K$ be the isotropy subgroup of $G$ at some point o of $\tilde{M}$. The subgroup $K$ is a maximal compact subgroup of $G$. Let $g$,

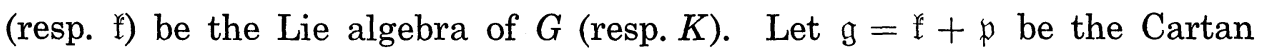
decomposition of $g$ corresponding to $\mathfrak{l}$. Then $\mathfrak{p}$ is identified with the tangent space of $\tilde{M}$ at $o$. Let $\mathfrak{a}$ be a maximal abelian subspace of $\mathfrak{p}, \mathfrak{a}^{*}$ its dual and $\mathfrak{a}_{c}^{*}$ the complexification of $\mathfrak{a}^{*}$. Then $\operatorname{rank}(\tilde{M}, g)=1$ means $\operatorname{dim} \mathfrak{a}=1$. Let $B$ be the Killing form of $g$. We assume the Riemannian metric $g$ on $\tilde{M}=G / K$ is induced by $g_{o}\left(X_{o}, Y_{o}\right)=B(X, Y), X, Y \in \mathfrak{p}$, where $X_{o}, Y_{o}$ are the tangent vectors of $\tilde{M}$ at $o=\{K\}$ corresponding to $X, Y$, respectively. For $\lambda \in \mathfrak{a}^{*}$, let $H_{\lambda} \in \mathfrak{a}$ be determined by $\lambda(H)=B\left(H_{\lambda}, H\right)$ for all $H \in \mathfrak{a}$. Put $(\lambda, \mu)=B\left(H_{\lambda}, H_{\mu}\right)$ for $\lambda, \mu \in \mathfrak{a}^{*}$. We fix an order on $\mathfrak{a}^{*}$ once and for all. Let $\Sigma$ be the set of all non-zero restricted roots of $(\mathfrak{g}, \mathfrak{a})$ and $\Sigma^{+}$the set of positive elements in $\Sigma$. For, $\alpha \in \Sigma$, let $\mathfrak{g}_{\alpha}=\{X \in \mathfrak{g} ;[H, X]=\alpha(H) X$ for all $H \in \mathfrak{a}$ \}. Let denote $m_{\alpha}=\operatorname{dim} g_{\alpha}$ for $\alpha \in \Sigma$, which is called the multiplicity of $\alpha$. Let $\delta=2^{-1} \sum_{\alpha \in \Sigma^{+}} m_{\alpha} \alpha$. Let $\mathfrak{n}=\sum_{\alpha \in \Sigma^{+}} \mathfrak{g}_{\alpha}$ and $N$ the connected subgroup of $G$ corresponding to $\mathfrak{n}$. Each $g \in G$ can be uniquely written as $g=\kappa(g) \exp (H(g)) n(g)$ where $\kappa(g) \in K, H(g) \in \mathfrak{a}$ and $n(g) \in N$. In case of rank one, the zonal spherical functions on $\tilde{M}$ mean the (complex valued) $K$-invariant eigen-functions of the Laplace-Beltrami operator $-\Delta$ of $(\tilde{M}, g)$ whose values at $o=\{K\}$ are 1 . These functions are exhausted by $\varphi_{\lambda}(g)=$

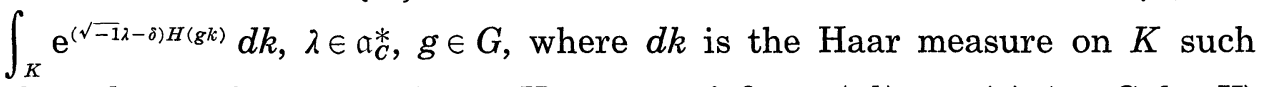
that the total measure is 1 . Here $\varphi_{\lambda}$ satisfies $\varphi_{\lambda}(g k)=\varphi_{\lambda}(g)(g \in G, k \in K)$ and hence it is regarded as a function on $\tilde{M}$. Notice that $\Sigma^{+}=\{\alpha,(2 \alpha)\}$ and $\delta=2^{-1}\left(m_{\alpha}+2 m_{2 \alpha}\right) \alpha$ since $\tilde{M}$ is of rank one. Let $H_{0} \in a$ be the element such that $\alpha\left(H_{0}\right)=1$ and hence $B\left(H_{0}, H_{0}\right)=2\left(m_{\alpha}+4 m_{2 \alpha}\right)$. For $t \in \boldsymbol{R}$, put $h_{t}=\exp \left(t H_{0}\right) \in A=\exp (\mathfrak{a})$. Then $t$ can be regarded as the coordinate on the one dimensional Lie group $A$. Put $x=-(\sinh (t))^{2}$. Since $\varphi_{k}\left(h_{t}\right)$ is an even function of $t$, it is written as $\varphi_{\lambda}\left(h_{t}\right)=g_{\lambda}(x)$. Then $g_{\lambda}$ satisfies

$$
x(x-1) \frac{d^{2}}{d x^{2}} g_{\lambda}+((a+b+1) x-c) \frac{d g_{\lambda}}{d x}=-a b g_{\lambda},
$$


where $a=4^{-1}\left(m_{\alpha}+2 m_{2 \alpha}+2 \sqrt{-1} \lambda\left(H_{0}\right)\right), \quad b=4^{-1}\left(m_{\alpha}+2 m_{2 \alpha}-2 \sqrt{-1} \lambda\left(H_{0}\right)\right)$ and $c=2^{-1}\left(m_{\alpha}+m_{2 \alpha}+1\right)$ (cf. [3] p. 301). Notice that $a+b, a b$ and $c>0$. Thus $g_{\lambda}(x)$ is the hypergeometric function $F(a, b, c ; x)$. Moreover, each $K-$ invariant function $F \in C^{2}(M-(o))$ satisfies

$$
\begin{aligned}
& -2^{-1}\left(m_{\alpha}+4 m_{2 \alpha}\right)(\Delta F)\left(k h_{t} \cdot o\right) \\
& \quad=x(x-1) \frac{d^{2} G}{d x^{2}}(x)+((a+b+1) x-c) \frac{d G}{d x}(x)
\end{aligned}
$$

$(t \neq 0, k \in K)$, where $G$ is the function defined by $F(t)=G(x)$ (cf. [3] p. 302). Thus we have

$$
2^{-1}\left(m_{\alpha}+4 m_{2 \alpha}\right) \Delta \varphi_{\lambda}=a b \varphi_{\lambda} .
$$

If $\lambda \in \mathfrak{a}^{*}, \varphi_{\lambda}$ is real valued and has the following asymptotic behavior:

$$
\lim _{t \rightarrow \infty}\left|\mathrm{e}^{t \delta\left(H_{0}\right)} \varphi_{\lambda}\left(h_{t}\right)-\left(c(\lambda) \mathrm{e}^{t \sqrt{-1} \lambda\left(H_{0}\right)}+c(-\lambda) \mathrm{e}^{-t \sqrt{-1} \lambda\left(H_{0}\right)}\right)\right|=0,
$$

where $c(\lambda)=\Gamma(c) \Gamma\left(\sqrt{-1} \lambda\left(H_{0}\right)\right) \Gamma(a)^{-1} \Gamma\left(m_{\alpha}+2+2 \sqrt{-1} \lambda\left(H_{0}\right)\right)^{-1}$ (cf. [3] p. 303).

Let $d g_{K}$ be the volume element of $(\tilde{M}, g)$. Then it is known (cf. [4] p. 381) that

$$
\int_{\tilde{M}} f(g \cdot o) d g_{K}=C \int_{-\infty}^{0} D(x) g(x) d x
$$

for every integrable $K$-invariant function $f$ on $\tilde{M}$ and $g(x)=f\left(h_{t} \cdot o\right), x=$ $-(\sinh (t))^{2}$. Here $C$ is a positive constant which does not depend on $f$ and $D(x)=(-x)^{2-1\left(m_{\alpha}+m_{2 \alpha}-1\right)}(1-x)^{2-1\left(m_{2 \alpha}-1\right)}$.

2.3. First, we notice that if $\lambda \in \mathfrak{a}^{*}, \lambda \neq 0$, then the function $\varphi_{\lambda}$ has zero points. For, since $|c(\lambda)|=|c(-\lambda)|, \overline{c(\lambda)}=c(-\lambda)$, we have by (2.6)

$$
\varphi_{\lambda}\left(h_{t}\right) \sim 2 \mathrm{e}^{-t \delta\left(H_{0}\right)}|c(\lambda)| \cos \left(t \lambda\left(H_{0}\right)+\arg (c(\lambda))\right), \quad \delta\left(H_{0}\right)>0,
$$

as $t \rightarrow \infty$. So let $-A_{\lambda}\left(0<A_{\lambda}<\infty\right)$ be the first zero point of $g_{\lambda}(x), x \leqq 0$. We consider also the function $f_{\lambda}$ defined by

$$
f_{\lambda}(x)= \begin{cases}g_{\lambda}(x)^{1+\varepsilon}, & -A_{\lambda} \leqq x \leqq 0, \\ 0, & -\infty<x<-A_{\lambda},\end{cases}
$$

where $0<\varepsilon<1$. The continuous function $f_{2}$ on $(-\infty, 0]$ has the following properties.

LEMma 2.3. ( $\left.1^{\prime}\right) f_{\lambda}$ belongs to $C^{1}(-\infty, 0]$ and the support of $f_{\lambda}$ is contained in the set $\left\{-A_{\lambda} \leqq x \leqq 0\right\},\left(2^{\prime}\right) f_{\lambda}^{\prime \prime}$ is continuous on $-\infty<x<0$ except 
$-A_{\lambda}, \quad\left(3^{\prime}\right) \quad f_{\lambda}^{\prime \prime}(x)=O\left(\left|x+A_{\lambda}\right|^{\mid-1}\right)$, so $f_{\lambda}^{\prime \prime} \in L^{1}(-\infty, 0]$, and $\left(4^{\prime}\right) L_{2}\left(f_{\lambda}\right)(x)+$ $(1+\varepsilon) a b f_{\lambda}(x) \geqq 0$, except $x=0,-A_{\lambda}$ for the differential operator $L_{2}$ on $(-\infty$, 0] defined by

$$
L_{2}=x(x-1) \frac{d^{2}}{d x^{2}}+((a+b+1) x-c) \frac{d}{d x} .
$$

Proof. $\left(1^{\prime}\right)$ and $\left(2^{\prime}\right)$ are clear. For $\left(3^{\prime}\right)$, we may show that $-A_{2}$ is the zero point of $g_{2}$ of first order. By the properties of the hypergeometric function $g_{\lambda}(x)=F(a, b, c ; x)$,

$$
\left((-x)^{c}(1-x)^{a+b-c+1} g_{2}^{\prime}\right)^{\prime}=-a b(-x)^{c-1}(1-x)^{a+b-c} g_{2} .
$$

Then $G(x)=(-x)^{c}(1-x)^{a+b-c+1} g_{2}^{\prime}$ satisfies $G^{\prime}(x)<0\left(-A_{2}<x<0\right)$ and $G^{\prime}(x)=0 \quad\left(x=0,-A_{\lambda}\right)$. Hence $G(x)>G(0)=0 \quad\left(-A_{\lambda} \leqq x<0\right)$, that is $g_{2}^{\prime}(x)>0\left(-A_{2} \leqq x<0\right)$. By (2.5),

$$
L_{2}\left(f_{\lambda}\right)(x)+(1+\varepsilon) a b f_{\lambda}(x)=(1+\varepsilon) \varepsilon x(x-1)\left(g_{\lambda}^{\prime}\right)^{2} g_{\lambda}^{\varepsilon-1} \geqq 0
$$

$\left(-A_{\lambda}<x<0\right)$, so $\left(4^{\prime}\right)$ holds.

Q.E.D.

Define a function $F_{\lambda}$ on $A$ by $F_{\lambda}\left(h_{t}\right)=f_{\lambda}(x), x=-(\sinh (t))^{2}$. Then it belongs to $C^{1}(A)$ and is an even function, that is $F_{\lambda}\left(h_{t}\right)=F_{\lambda}\left(h_{-t}\right)$. Hence it can be extended to $\tilde{M}$ uniquely as a $K$-invariant function, denoted by the same letter $F_{\lambda}$. It satisfies the following properties.

Lemma 2.4. $F_{2}$ belongs to $C^{1}(\tilde{M})$ and $C^{2}\left(\tilde{M}-\gamma_{2}\right)$ where $\gamma_{2}=\left\{k h_{t} \cdot o\right.$; $\left.k \in K,-(\sinh (t))^{2}=0,-A_{\lambda}\right\}$, and the support of $F_{\lambda}$ is contained in the set $\left\{k h_{t} \cdot o ; k \in K,-A_{\lambda} \leqq-(\sinh (t))^{2}\right\} . \quad$ Moreover

$$
2^{-1}\left(m_{\alpha}+4 m_{2 \alpha}\right)\left(\Delta F_{\lambda}\right)\left(k h_{t} \cdot o\right)=-\left(L_{2} f_{\lambda}\right)(x)
$$

$(t \neq 0, k \in K)$ and $\Delta F_{\lambda} \in L^{1}(\tilde{M})$,

$$
2^{-1}\left(m_{\alpha}+4 m_{2 \alpha}\right) \Delta F_{\lambda} \leqq(1+\varepsilon) a b F_{\lambda} \quad \text { on } \tilde{M}-\gamma_{2},
$$

where $L^{1}(\tilde{M})$ is the space of integrable functions on $\tilde{M}$ with respect to the volume element $d g_{K}$ in (2.7).

Proof. (2.8) follows from (2.7), (2.5) and Lemma 2.3. The remainds are immediate from Lemma 2.3.

Q.E.D.

Due to Lemma 2.3, there exists a sequence $\left\{F_{\lambda, m}\right\}_{m=1}^{\infty}$ of smooth even functions on $A$ such that $\left(5^{\prime}\right) F_{\lambda, m}\left(h_{t}\right)=F_{\lambda}\left(h_{t}\right) \quad\left(|t| \leqq t_{o} / 2\right)$ and $F_{\lambda, m}\left(h_{t}\right)=0$ $\left(|t| \geqq 2 t_{o}\right)$, where $t_{o}>0$ is given by $-\left(\sinh \left(t_{o}\right)\right)^{2}=-A_{\lambda},\left(6^{\prime}\right) F_{\lambda, m}\left(\right.$ resp. $\left.F_{\lambda, m}^{\prime}\right)$ 
converges to $F_{\lambda}$ (resp. $\left.F_{\lambda}^{\prime}\right)$ uniformly on $A$ as $m \rightarrow \infty$, and (7')

$$
\lim _{m \rightarrow \infty} \int_{-\infty}^{\infty}\left|F_{\lambda, m}^{\prime \prime}\left(h_{t}\right)-F_{\lambda}^{\prime \prime}\left(h_{t}\right)\right| d t=0
$$

where $F_{\lambda}^{\prime}$ etc. means the differential of $F_{\lambda}$ with respect to $t$. The functions $F_{\lambda, m}$ can be extended as $K$-invariant $C^{\infty}$ functions on $\tilde{M}$, denoted by the same letter $F_{\lambda, m}$. Then the support of $F_{\lambda, m}$ is contained in the set $\left\{k h_{t} \cdot o\right.$; $\left.k \in K,|t| \leqq 2 t_{o}\right\}, F_{\lambda, m}$ converges to $F_{\lambda}$ uniformly on $\tilde{M}$ and

$$
\lim _{m \rightarrow \infty} \int_{\tilde{M}}\left|\Delta F_{\lambda, m}-\Delta F_{\lambda}\right| d g_{K}=0
$$

by $\left(5^{\prime}\right),\left(6^{\prime}\right),\left(7^{\prime}\right),(2.5)$ and $(2.7)$.

\section{§3. Proof of Theorem A}

3.1. In this section, we preserve the notations in 2.1 and introduction. Let $\pi$ denote the projection of $\boldsymbol{R}^{n}$ onto $M_{\Gamma}$. For $\gamma \in \Gamma$, let $\tau_{\gamma}$ be the action of $\gamma$ on $\boldsymbol{R}^{n}$. The Laplace-Beltrami operator $-\Delta_{\Gamma}$ on $M_{\Gamma}$ satisfies $\Delta(f \circ \pi)=$ $\left(\Delta_{T} f\right) \circ \pi$ for twice differentiable functions $f$ on $M_{\Gamma}$. The volume element on $M_{\Gamma}$ induced by $d x$ is denoted by $d \omega$. Let $\mathscr{F}$ be the fundamental domain in $\boldsymbol{R}^{n}$ for $\Gamma$, that is $\mathscr{F}=\left\{x \in \boldsymbol{R}^{n} ;|x| \leqq\left|x-\tau_{r} \cdot 0\right|\right.$ for all $\left.\gamma \in \Gamma\right\}$. It is known (cf. [7]) that

$$
\boldsymbol{R}^{n}=\bigcup_{r \in \Gamma} \tau_{r} \cdot \mathscr{F} \quad \text { and } \quad \tau_{r} \cdot \mathscr{F} \cap \mathscr{F}
$$

has measure 0 for every $\gamma \in \Gamma, \gamma \neq 1$.

Now since the functions $F$ and $F_{m}$ have the compact supports, we can define the $\Gamma$-invariant functions $\theta$ and $\theta_{m}$ on $\boldsymbol{R}^{n}$ by

$$
\theta=\sum_{r \in \Gamma} F \circ \tau_{r}, \quad \theta_{m}=\sum_{r \in \Gamma} F_{m} \circ \tau_{r} .
$$

Then there exist functions $\varphi$ and $\varphi_{m}$ on $M_{\Gamma}$ such that $\varphi \circ \pi=\theta, \varphi_{m} \circ \pi=\theta_{m}$. These functions have the following properties:

LemMa 3.1. (1) The function $\varphi$ belongs to $C^{1}\left(M_{\Gamma}\right)$ and $C^{2}\left(M_{\Gamma}-\pi\left(\gamma_{1}\right)\right)$, and $\Delta_{\Gamma} \varphi$ belongs to $L^{1}\left(M_{\Gamma}\right)$, (2) $\Delta_{\Gamma} \varphi \leqq(1+\varepsilon) p^{2} \varphi$ on $M_{\Gamma}-\pi\left(\gamma_{1}\right)$, and (3) $\varphi_{m} \in C^{\infty}\left(M_{\Gamma}\right)$ converges to $\varphi$ uniformly on $M_{\Gamma}$ as $m \rightarrow \infty$, and

$$
\lim _{m \rightarrow \infty} \int_{M_{\Gamma}}\left|\Delta_{\Gamma} \varphi_{m}-\Delta_{\Gamma} \varphi\right| d \omega=0 \text {. }
$$

Moreover (4) 


$$
\lim _{m \rightarrow \infty} \int_{M_{\Gamma}} \varphi_{m}\left(\Delta_{\Gamma} \varphi_{m}\right) d \omega=\int_{M_{\Gamma}} \varphi\left(\Delta_{\Gamma} \varphi\right) d \omega
$$

Proof. (1), (2) and (3) follow from Lemma 2.2 and (2.4). The inequality

$$
\begin{aligned}
& \left|\int_{M_{\Gamma}} \varphi_{m}\left(\Delta_{\Gamma} \varphi_{m}\right) d \omega-\int_{M_{\Gamma}} \varphi\left(\Delta_{\Gamma} \varphi\right) d \omega\right| \\
& \quad \leqq\left\|\Delta_{\Gamma} \varphi_{m}-\Delta_{\Gamma} \varphi\right\|_{L^{1}\left(M_{\Gamma}\right)} \cdot \sup _{M_{\Gamma}}\left|\varphi_{m}\right|+\left\|\Delta_{\Gamma} \varphi\right\|_{L^{1}\left(M_{\Gamma}\right)} \cdot \sup _{M_{\Gamma}}\left|\varphi_{m}-\varphi\right|,
\end{aligned}
$$

together with (3), implies (4).

Notice that

$$
\begin{gathered}
\int_{M_{\Gamma}} \varphi d \omega=V_{n-1} p^{-n} \int_{0}^{j_{n / 2-1}} \psi(r)^{1+\varepsilon} r^{n-1} d r, \\
\int_{M_{\Gamma}} \varphi^{2} d \omega \geqq V_{n-1} p^{-n} \int_{0}^{j_{n / 2-1}} \psi(r)^{2(1+\varepsilon)} r^{n-1} d r,
\end{gathered}
$$

where $V_{n-1}=2 \pi^{n / 2} \Gamma(n / 2)^{-1}$ is the total measure of the unit sphere $S^{n-1}$ with respect to the measure induced by the volume element $d x$ on $R^{n}$. In fact, by (3.1) and the definitions of $\varphi, \theta$ and $F$, we have

$$
\begin{aligned}
\int_{M_{\Gamma}} \varphi d \omega & =\int_{\mathscr{F}} \theta d x \\
& =\sum_{r \in \Gamma} \int_{\mathscr{F}}\left(F \circ \tau_{\gamma}\right) d x \\
& =\int_{\cup_{r} \in \Gamma^{\tau_{\gamma}} \cdot \mathscr{F}} F d x \\
& =\int_{R^{n}} F d x \\
& =V_{n-1} p^{-n} \int_{0}^{j_{n / 2-1}} \psi(r)^{1+\varepsilon} r^{n-1} d r .
\end{aligned}
$$

(3.2) follows from the inequality for the integrand:

$$
\theta^{2}=\sum_{r, r^{\prime} \in \Gamma}\left(F \circ \tau_{r}\right)\left(F \circ \tau_{\gamma^{\prime}}\right) \geqq \sum_{r \in \Gamma}\left(F \circ \tau_{r}\right)^{2},
$$

which follows from $F \geqq 0$.

3.2. It is known (cf. [1] p. 186) that the least positive eigenvalue $\lambda_{1}(\Gamma)$ of $\Delta_{\Gamma}$ satisfies the inequality

$$
\int_{M_{\Gamma}} \eta\left(\Delta_{\Gamma} \eta\right) d \omega \geqq \lambda_{1}(\Gamma) \int_{M_{\Gamma}} \eta^{2} d \omega
$$


for all $\eta \in C^{\infty}\left(M_{\Gamma}\right)$ such that

$$
\int_{M_{\Gamma}} \eta d \omega=0
$$

We apply (3.4) for $\eta=\varphi_{m}-\alpha_{m}$, where

$$
\alpha_{m}=\operatorname{vol}\left(M_{\Gamma}\right)^{-1} \int_{M_{\Gamma}} \varphi_{m} d \omega
$$

Then we have

$$
\int_{M_{\Gamma}} \varphi_{m}\left(\Delta_{\Gamma} \varphi_{m}\right) d \omega \geqq \lambda_{1}(\Gamma)\left[\int_{M_{\Gamma}} \varphi_{m}^{2} d \omega-\operatorname{vol}\left(M_{\Gamma}\right)^{-1}\left(\int_{M_{\Gamma}} \varphi_{m} d \omega\right)^{2}\right] .
$$

As $m \rightarrow \infty$, we have

$$
\int_{M_{\Gamma}} \varphi\left(\Delta_{\Gamma} \varphi\right) d \omega \geqq \lambda_{1}(\Gamma)\left[\int_{M_{\Gamma}} \varphi^{2} d \omega-\operatorname{vol}\left(M_{\Gamma}\right)^{-1}\left(\int_{M_{\Gamma}} \varphi d \omega\right)^{2}\right]
$$

by (3) and (4) in Lemma 3.1. Since $\pi\left(\gamma_{1}\right)$ has measure 0, we have

$$
\int_{M_{\Gamma}} \varphi\left(\Delta_{\Gamma} \varphi\right) d \omega \leqq(1+\varepsilon) p^{2} \int_{M_{\Gamma}} \varphi^{2} d \omega
$$

by (2) in Lemma 3.1. Then, by (3.5) and (3.6),

$$
\lambda_{1}(\Gamma)\left[1-\operatorname{vol}\left(M_{\Gamma}\right)^{-1}\left(\int_{M_{\Gamma}} \varphi d \omega\right)^{2}\left(\int_{M_{\Gamma}} \varphi^{2} d \omega\right)^{-1}\right] \leqq(1+\varepsilon) p^{2} .
$$

Hence, together with (3.2) and (3.3), we have

$$
\begin{aligned}
& \lambda_{1}(\Gamma)\left[1-p^{-n}\right. V_{n-1} \operatorname{vol}\left(M_{\Gamma}\right)^{-1}\left(\int_{0}^{j_{n / 2}-1} \psi(r)^{1+\varepsilon} r^{n-1} d r\right)^{2} \\
&\left.\times\left(\int_{0}^{j n / 2-1} \psi(r)^{2(1+\varepsilon)} r^{n-1} d r\right)^{-1}\right] \leqq(1+\varepsilon) p^{2}
\end{aligned}
$$

Letting $\varepsilon \rightarrow 0$, we obtain

Proposition 3.1. Under the above situation, we have

$$
\begin{aligned}
\lambda_{1}(\Gamma) \leqq & \inf _{p>0}\left\{p^{2}\left[1-V_{n-1} K_{n} \operatorname{vol}\left(M_{\Gamma}\right)^{-1} p^{-n}\right]^{-1} ;\right. \\
& \left.1-V_{n-1} K_{n} \operatorname{vol}\left(M_{\Gamma}\right)^{-1} p^{-n}>0\right\}
\end{aligned}
$$

where

$$
K_{n}=\left(\int_{0}^{j n / 2-1} \psi(r) r^{n-1} d r\right)^{2}\left(\int_{0}^{j n / 2-1} \psi(r)^{2} r^{n-1} d r\right)^{-1}
$$

and 


$$
V_{n-1}=2 \pi^{n / 2} \Gamma\left(\frac{n}{2}\right)^{-1}
$$

3.3. We calculate the right hand side of (3.7). Since

$$
\psi(r)=\Gamma\left(\frac{n}{2}\right)\left(\frac{r}{2}\right)^{1-n / 2} J_{n / 2-1}(r),
$$

we have

$$
K_{n}=\left(\int_{0}^{j_{n / 2-1}} J_{n / 2-1}(r) r^{n / 2} d r\right)^{2}\left(\int_{0}^{j n / 2-1} J_{n / 2-1}(r)^{2} r d r\right)^{-1} .
$$

Since the derivative of $J_{n / 2}(r) r^{n / 2}$ (resp. $\left.\left(r^{2} / 2\right)\left(J_{n / 2-1}(r)^{2}-J_{n / 2-2}(r) J_{n / 2}(r)\right)\right)$ is $J_{n / 2-1}(r) r^{n / 2}$ (resp. $\left.J_{n / 2-1}(r)^{2} r\right)$ (cf. [7] p. 189), we have

$$
\begin{aligned}
K_{n}= & \left(J_{n / 2}\left(j_{n / 2-1}\right)\left(j_{n / 2-1}\right)^{n / 2}\right)^{2}\left(\frac{1}{2}\left(j_{n / 2-1}\right)^{2}(-1) J_{n / 2-2}\left(j_{n / 2-1}\right) J_{n / 2}\left(j_{n / 2-1}\right)\right)^{-1}, \\
= & \left(J_{n / 2}\left(j_{n / 2-1}\right)\left(j_{n / 2-1}\right)^{n / 2}\right)^{2}\left(\frac{1}{2}\left(j_{n / 2-1}\right)^{2} J_{n / 2}\left(j_{n / 2-1}\right)^{2}\right)^{-1} \\
& \quad\left(\text { by } J_{n / 2}\left(j_{n / 2-1}\right)+J_{n / 2-2}\left(j_{n / 2-1}\right)=0 \text { (cf. [7] p. 158)) },\right. \\
= & 2\left(j_{n / 2-1}\right)^{n-2} .
\end{aligned}
$$

Put

$$
G(p)=p^{2}\left(1-V_{n-1} K_{n} \operatorname{vol}\left(M_{\Gamma}\right)^{-1} p^{-n}\right)^{-1}
$$

and

$$
p_{0}=\left(2^{-1}(2+n) V_{n-1} K_{n} \operatorname{vol}\left(M_{\Gamma}\right)^{-1}\right)^{1 / n} .
$$

If $1-V_{n-1} K_{n} \operatorname{vol}\left(M_{\Gamma}\right)^{-1} p^{-n}>0$, then

$$
G^{\prime}(p)<0\left(p<p_{0}\right), \quad G^{\prime}(p)>0\left(p>p_{0}\right) \text { and } G^{\prime}\left(p_{0}\right)=0 .
$$

So we have

$$
\begin{aligned}
\inf _{p>0}\{G(p) & \left.; 1-V_{n-1} K_{n} \operatorname{vol}\left(M_{\Gamma}\right)^{-1} p^{-n}>0\right\}=G\left(p_{0}\right) \\
= & 2^{-2 / n} n^{-1}(2+n)^{2 / n+1}\left(V_{n-1} K_{n} \operatorname{vol}\left(M_{\Gamma}\right)^{-1}\right)^{2 / n} \\
= & n^{-1}(2+n)^{2 / n+1}\left(\frac{2 \pi^{n / 2}}{\Gamma(n / 2)}\right)^{2 / n}\left(j_{n / 2-1}\right)^{2-4 / n} \operatorname{vol}\left(M_{\Gamma}\right)^{-2 / n}
\end{aligned}
$$

Thus Theorem A is proved.

\section{§4. Proof of Theorem B}

4.1. In this section, we preserve the notations in $2.2,2.3$ and introduction. Theorem B will be proved by the same way as Theorem A. Let 
$\pi$ denote the projection of $\tilde{M}$ onto $M_{\Gamma}$. For $\gamma \in \Gamma$, let $\tau_{\gamma}$ be the action of $\gamma$ on $\tilde{M}$. The Laplace-Beltrami operator $-\Delta_{T}$ on $M$ satisfies $\Delta(f \circ \pi)=$ $\left(\Delta_{T} f\right) \circ \pi$ for all twice differentiable functions $f$ on $M_{\Gamma}$. The volume element on $M_{\Gamma}$ induced by $d g_{K}$ is denoted by $d \omega$. Let $\mathscr{F}$ be the fundamental domain in $\tilde{M}$ for $\Gamma$, that is $\mathscr{F}=\left\{g \cdot o \in \tilde{M} ; r(g \cdot o, o) \leqq r\left(g \cdot o \cdot \tau_{r} \cdot o\right)\right.$ for all $\left.\gamma \in \Gamma\right\}$ where $r(\cdot, \cdot)$ is the distance function on $(\tilde{M}, g)$. It is known (for example, cf. [2] ) that

$$
\tilde{M}=\bigcup_{r \in \Gamma} \tau_{r} \mathscr{F} \quad \text { and } \quad \tau_{r} \mathscr{F} \cap \mathscr{F}
$$

has measure 0 for all $\gamma \in \Gamma, \gamma \neq 1$.

Since $F_{\lambda}$ and $F_{\lambda, m}$ have the compact supports, we define the $\Gamma$-invariant functions $\theta_{\lambda}$ and $\theta_{\lambda, m}$ on $\tilde{M}$ by

$$
\theta_{\lambda}=\sum_{r \in \Gamma} F_{\lambda} \circ \tau_{r}, \quad \theta_{\lambda, m}=\sum_{r \in \Gamma} F_{\lambda, m} \circ \tau_{r} .
$$

Then there exist functions $\varphi_{\lambda}$ and $\varphi_{\lambda, m}$ on $M_{\Gamma}$ such that $\varphi_{\lambda} \circ \pi=\theta_{\lambda}$ and $\varphi_{\lambda, m} \circ \pi=\theta_{\lambda, m}$. These functions have the following properties.

Lemma 4.1. (1) The function $\varphi_{\lambda}$ belongs to $C^{1}\left(M_{\Gamma}\right)$ and $C^{2}\left(M_{\Gamma}-\pi\left(\gamma_{2}\right)\right)$, and $\Delta_{\Gamma} \varphi_{\lambda}$ belongs to $L^{1}\left(M_{\Gamma}\right)$, (2) $2^{-1}\left(m_{\alpha}+4 m_{2 \alpha}\right) \Delta_{\Gamma} \varphi_{\lambda} \leqq(1+\varepsilon) a b \varphi_{\lambda}$ on $M_{\Gamma}-$ $\pi\left(\gamma_{2}\right)$, and (3) $\varphi_{\lambda, m} \in C^{\infty}\left(M_{\Gamma}\right)$ converges to $\varphi_{\lambda}$ uniformly on $M_{\Gamma}$ as $m \rightarrow \infty$ and

$$
\lim _{m \rightarrow \infty} \int_{M_{\Gamma}}\left|\Delta_{\Gamma} \varphi_{\lambda, m}-\Delta_{\Gamma} \varphi_{\lambda}\right| d \omega=0 .
$$

Moreover (4)

$$
\lim _{m \rightarrow \infty} \int_{M_{\Gamma}} \varphi_{\lambda, m}\left(\Delta_{\Gamma} \varphi_{\lambda, m}\right) d \omega=\int_{M_{\Gamma}} \varphi_{\lambda}\left(\Delta_{\Gamma} \varphi_{\lambda}\right) d \omega
$$

Proofs are similar to Lemma 3.1.

Notice that

$$
\begin{gathered}
\int_{M_{\Gamma}} \varphi_{\lambda} d \omega=\int_{\tilde{M}} F_{\lambda} d g_{K}=C \int_{-A_{\lambda}}^{0} D(x) g_{\lambda}(x)^{1+\varepsilon} d x, \\
\int_{M_{\Gamma}} \varphi_{\lambda}^{2} d \omega \geqq C \int_{-A_{\lambda}}^{0} D(x) g_{\lambda}(x)^{2(1+\varepsilon)} d x .
\end{gathered}
$$

Then, due to (4.2), (4.3) and Lemma 4.1, we have the following proposition by the similar manner to Proposition 3.1 .

Proposition 4.1. Under the above assumption, we obtain 


$$
\lambda_{1}(\Gamma)\left[1-C \operatorname{vol}\left(M_{\Gamma}\right)^{-1} K_{\lambda}\right] \leq 2\left(m_{\alpha}+4 m_{2 \alpha}\right)^{-1} a b,
$$

where

$$
K_{\lambda}=\left(\int_{-A_{\lambda}}^{0} D(x) g_{\lambda}(x) d x\right)^{2}\left(\int_{-A_{\lambda}}^{0} D(x) g_{\lambda}(x)^{2} d x\right)^{-1},
$$

the constant $C$ and the function $D(x)$ are the ones in (2.7).

4.2. We prove Theorem B due to Proposition 4.1. We fix any $\lambda \in \mathfrak{a}^{*}$, $\lambda \neq 0$. For a discrete subgroup $\Gamma$ of $G$ with sufficiently large vol $\left(M_{\Gamma}\right)$ such that vol $\left(M_{\Gamma}\right)>C K_{\lambda}$, we have, by Proposition 4.1,

$$
\lambda_{1}(\Gamma) \leqq 2\left(m_{\alpha}+4 m_{2 \alpha}\right)^{-1} a b\left[1-C \operatorname{vol}\left(M_{\Gamma}\right)^{-1} K_{\lambda}\right]^{-1} .
$$

Hence, by the definition of $a$ and $b$, we have

$$
\begin{aligned}
\lim _{\text {vol }\left(M \Gamma_{T}\right) \rightarrow \infty} \lambda_{1}(\Gamma) & \leqq 2\left(m_{\alpha}+4 m_{2 \alpha}\right)^{-1} a b \\
& =\frac{1}{8}\left(m_{\alpha}+4 m_{2 \alpha}\right)^{-1}\left(\left(m_{\alpha}+4 m_{2 \alpha}\right)^{2}+4 \lambda\left(H_{0}\right)^{2}\right)
\end{aligned}
$$

for every $\lambda \in \mathfrak{a}^{*}, \lambda \neq 0$. So we have

$$
\lim _{\operatorname{vol}\left(M \Gamma^{\prime}\right) \rightarrow \infty} \lambda_{1}(\Gamma) \leqq \frac{1}{8}\left(m_{\alpha}+4 m_{2 \alpha}\right)^{-1}\left(m_{\alpha}+2 m_{2 \alpha}\right)^{2} .
$$

Here $B\left(H_{0}, H_{0}\right)=2\left(m_{\alpha}+4 m_{2 \alpha}\right)$ implies that the right hand side of the above inequality coincides with $|\delta|^{2}=(\delta, \delta)$. Thus Theorem B is proved.

\section{§5. Supremum of $\mathbf{L}^{2}$ spectrum}

For a complete orientable Riemannian manifold $(M, g)$ (not necessarily compact), consider

$$
\sigma(M, g)=\inf _{\varphi \in C_{o}^{\infty}(M)} \frac{\int_{M}\left(\Delta_{g} \varphi\right) \varphi d v_{g}}{\int_{M} \varphi^{2} d v_{g}}
$$

where $C_{o}^{\infty}(M)$ is the space of all real valued $C^{\infty}$ functions on $M$ with compact support, $-\Delta_{g}$ is the Laplace-Beltrami operator of $(M, g)$ acting on smooth functions on $M$, and $d v_{g}$ is the volume element of $(M, g)$ (cf. [11]). Then $\sigma(M, g) \geqq 0$ and it is called the supremum of the $L^{2}$ spectrum of $(M, g)$ (cf. [11]). Since the operator $\Delta_{g}$ is a real symmetric operator, we notice that

$$
\sigma(M, g)=\inf _{\varphi \in C_{0}^{\infty}(M)} c \frac{\int_{M}\left(\Delta_{g} \varphi\right) \bar{\varphi} d v_{g}}{\int_{M} \varphi \bar{\varphi} d v_{g}}
$$


where $C_{o}^{\infty}(M)^{C}$ is the space of all complex valued $C^{\infty}$ functions on $M$ with compact support, and $\bar{\varphi}(x), x \in M$, is the complex conjugate of $\varphi(x)$.

In this section, we calculate $\sigma(M, g)$ when $(M, g)$ is a Riemannian symmetric space of non-compact type of rank one. We preserve the notations in $\S 2$.

Proposition 5.1. Let $(\tilde{M}, g)$ be a Riemannian symmetric space of noncompact type (not necessarily of rank one). We normalize $g$ in such a way that it is induced by the Killing form of the Lie algebra g of the connected component $G$ of the Lie group of all isometries of $(\tilde{M}, g)$. Then we have

$$
\sigma(\tilde{M}, g) \geqq|\delta|^{2},
$$

where $|\delta|$ is the norm of $\delta=\frac{1}{2} \sum_{\alpha \in \Sigma}+m_{\alpha} \alpha$ by the inner product induced from the Killing form as in $\S 2$.

Proof. It holds (cf. [10]) that

$$
\int_{\tilde{M}}\left(\Delta_{g} f\right) \bar{f} d g_{K}=C \int_{a^{*} \times K / Z_{K}(a)}\left(\Delta_{g} f\right)^{\sim}(\lambda, \dot{k}) \overline{\tilde{f}(\lambda, \dot{k})}|c(\lambda)|^{-2} d \lambda d \dot{k},
$$

for each $f \in C_{o}^{\infty}(\tilde{M})$. Here $C$ is a positive constant, not depending on $f$, $Z_{K}(\mathfrak{a})$ is the centralizer of $\mathfrak{a}$ in $K, \tilde{f}(\lambda, \dot{k})\left(\lambda \in \mathfrak{a}^{*}, \dot{k} \in K / Z_{K}(\mathfrak{a})\right)$ is the Fourier transform of $f$ defined by

$$
\tilde{f}(\lambda, \dot{k})=\int_{\tilde{M}} f(g \cdot o) e^{-(\sqrt{-1} \lambda+\delta) H\left(g^{-1} k\right)} d g_{K},
$$

$d \lambda$ is the Euclidean measure on $\mathfrak{a}^{*}$, and $d \dot{k}$ is the measure on $K / Z_{K}(\mathfrak{a})$ induced by the Haar measure $d k$ on $K$ (cf. [10]). Since $\left(\Delta_{g} f\right)^{\sim}(\lambda, \dot{k})=$ $\left(|\lambda|^{2}+|\delta|^{2}\right) \tilde{f}(\lambda, \dot{k})$ (cf. [12] p. 92, [13] p. 458),

the right hand side of (5.1)

$$
\begin{aligned}
& \geqq|\delta|^{2} C \int_{\mathrm{a}^{*} \times K / Z_{K}(\mathrm{a})}|\tilde{f}(\lambda, \dot{k})|^{2}|c(\lambda)|^{-2} d \lambda d \dot{k} \\
& =|\delta|^{2} \int_{\tilde{M}} f \bar{f} d g_{K} .
\end{aligned}
$$

Thus we have $\sigma(\tilde{M}, g) \geqq|\delta|^{2}$.

Q.E.D.

In particular, when $(\tilde{M}, g)$ is of rank one, the following theorem holds.

Theorem C. Let $(\tilde{M}, g)$ be a Riemannian symmetric space of noncompact type of rank one. We normalize g as in Proposition 5.1. Then we have 


$$
\sigma(\tilde{M}, g)=|\delta|^{2} .
$$

Proof. We may prove $\sigma(\tilde{M}, g) \leqq|\delta|^{2}$. We use the notations in 2.3. Since the supports of $\dot{F}_{\lambda}$ and $F_{\lambda, m}, m=1,2, \cdots$ are contained in the set $\left\{k h_{t} \cdot o ; k \in K, 0 \leqq t \leqq 2 t_{o}\right\}$, and $F_{\lambda, m}$ converges to $F_{\lambda}$ uniformly on $\tilde{M}$,

$$
\lim _{m \rightarrow \infty} \int_{\tilde{M}} F_{\lambda, m}^{2} d g_{K}=\int_{\tilde{M}} F_{\lambda}^{2} d g_{K} .
$$

Moreover we have

$$
\lim _{m \rightarrow \infty} \int_{\tilde{M}}\left(\Delta_{g} F_{\lambda, m}\right) F_{\lambda, m} d g_{K}=\int_{\tilde{\mu}}\left(\Delta_{g} F_{\lambda}\right) F_{\lambda} d g_{K} .
$$

In fact, it follows from the inequality

$$
\begin{aligned}
& \left|\int_{\tilde{M}}\left(\Delta_{g} F_{\lambda, m}\right) F_{\lambda, m} d g_{K}-\int_{\tilde{M}}\left(\Delta_{g} F_{\lambda}\right) F_{\lambda} d g_{K}\right| \\
& \quad \leqq\left\|\Delta_{g} F_{\lambda, m}-\Delta_{g} F_{\lambda}\right\|_{L^{1}(\tilde{M})} \sup _{\tilde{M}}\left|F_{\lambda, m}\right|+\left\|\Delta_{g} F_{\lambda}\right\|_{L^{1}(\tilde{M})} \sup _{\tilde{M}}\left|F_{\lambda, m}-F_{\lambda}\right|,
\end{aligned}
$$

(2.10), (2.8) and Lemma 2.4.

Thus we have

$$
\sigma(\tilde{M}, g) \int_{\tilde{M}} F_{\lambda}^{2} d g_{K} \leqq \int_{\tilde{M}}\left(\Delta_{g} F_{\lambda}\right) F_{\lambda} d g_{K},
$$

by (5.2), (5.3) and the definition of $\sigma(\tilde{M}, g)$. Moreover we estimate

$$
\text { the right hand side of (5.4) }
$$

$$
\leqq 2\left(m_{\alpha}+4 m_{2 \alpha}\right)^{-1}(1+\varepsilon) a b \int_{\tilde{M}} F_{\lambda}^{2} d g_{K},
$$

due to (2.9). Then

$$
\sigma(\tilde{M}, g) \leqq 2\left(m_{\alpha}+4 m_{2 \alpha}\right)^{-1}(1+\varepsilon) a b,
$$

for every $0<\varepsilon<1$ and $0 \neq \lambda \in \mathfrak{a}^{*}$. Thus we have $\sigma(\tilde{M}, g) \leqq|\delta|^{2}$. Q.E.D.

Remark. Due to 2.1, it is proved by the similar way to Theorem C, that

$$
\sigma\left(\boldsymbol{R}^{n}, g\right)=0,
$$

where $\left(\boldsymbol{R}^{n}, g\right)$ is the standard Euclidean space. 


\section{REFERENCES}

[1] M. Berger, P. Gauduchon and E. Mazet, Le spectre d'une variété riemannienne, Lecture note in Math., 194, Springer-Verlag, New York, 1971.

[2] R. Gangolli, Asymptotic behavior of spectra of compact quotients of certain symmetric spaces, Acta Math., 12 (1968), 151-192.

[ 3 ] Harish-Chandra, Spherical functions on a semisimple Lie group, I, Amer. J. Math., 80 (1958), 241-310.

[4] S. Helgason, Differential geometry and symmetric spaces, Academic Press, New York, 1962.

[ 5 ] H. Huber, Über den ersten Eigenwert des Laplace-Operators auf kompakten Riemannschen Flachen, Commet. Math. Helv., 49 (1974), 251-259.

[6] J. Milnor and D. Husemoller, Symmetric bilinear forms, Springer-Verlag, New York, 1973.

[7] S. Moriguchi, K. Udagawa and S. Hitotsumatsu, Mathematical formulas, III (in Japanese), Iwanami Shoten, Tokyo, 1960.

[ 8 ] T. Sunada, Spectrum of a compact flat manifold, Commet. Math. Helv., 53 (1978), 613-621.

[9] N. J. Vilenkin, Special functions and the theory of group representations, Trans. Math. Mono., Amer. Math. Soc., 22 (1968).

[10] G. Warner, Harmonic analysis on semi-simple Lie groups, II, Springer-Verlag, New York, 1972.

[11] S. Y. Cheng, Eigenvalue comparison theorems and its geometric applications, Math. Z., 143 (1975), 289-297.

[12] S. Helgason, A duality for symmetric spaces with applications to group representations, Advance Math., 5 (1970), 1-154.

[13] - The surjectivity of invariant differential operators on symmetric spaces, I, Ann. Math., 98 (1973), 451-479.

Department of Mathematics

College of General Education

Tohoku University 